\title{
Evidence of learning and memory in the juvenile dwarf cuttlefish Sepia bandensis
}

\author{
Jessica Bowers $^{1} \cdot$ Tahirah Nimi $^{1} \cdot$ Jack Wilson $^{1} \cdot$ Shannon Wagner ${ }^{1} \cdot$ Dragoş Amarie $^{2} \cdot$ Vinoth Sittaramane $^{1}$ \\ Published online: 4 June 2020 \\ (C) The Psychonomic Society, Inc. 2020
}

\begin{abstract}
Measuring behavior in the form of numerical data is difficult, especially for studies involving complex actions. DanioVision is a closed-chamber system that utilizes subject tracking to comprehensively record behavior, while also mitigating the influence of environmental conditions. We used DanioVision to record activity of juvenile dwarf cuttlefish (Sepia bandensis) during the inaccessible prey (IP) procedure, a memory experiment in which cuttlefish learn to inhibit capture attempts towards inaccessible prey. By quantifying total movement and orientation of the body, we found that cuttlefish show memory by selectively inhibiting tentacle strikes without reducing total movement, or orientation towards the prey. We show that DanioVision can be used to assess multiple components of dynamic responses that are not measurable by direct observation alone and provide new evidence that strike inhibition is the product of learning, and not motor fatigue.
\end{abstract}

Keywords Cephalopods $\cdot$ Learning $\cdot$ Memory, long-term $\cdot$ Sepia

\section{Introduction}

The cephalopods - including octopus, squid, and cuttlefish offer a unique opportunity to study memory in a complex invertebrate system. Cephalopods are suitable behavioral models, because they share many characteristics with vertebrates, including a centralized nervous system, and highly developed sensory systems, such as the visual system (Boycott 1961; Mather \& Kuba, 2013). Cuttlefish demonstrate advanced types of memory not documented in many invertebrate taxa, evidenced by spatial learning (Alves et al. 2007; Grasso \& Basil, 2009; Purdy et al., 1999), and various visual learning tasks (Lin \& Chiao, 2017; Zylinksi et al., 2012), episodic memory (Jozet-Alves et al., 2013), and source memory (Billard et al., 2020).

Cuttlefish utilize spatial learning to solve mazes, (Alves et al., 2007), navigate towards a reward (Purdy et al., 1999), and discriminate between vertical and horizontal cues (Scatà et al., 2016). Spatial learning is complex because many external

Vinoth Sittaramane

vsittaramane@georgiasouthern.edu

1 Department of Biology, Georgia Southern University, 4324 Old Register Rd, Statesboro, GA 30460, USA

2 Department of Physics and Astronomy, Georgia Southern University, 65 Georgia Ave, Statesboro, GA 30460, USA cues are incorporated into a "cognitive map" that represents the subject's location in the environment (Manns \& Eichenbaum, 2009). Visual learning in cuttlefish is shaped by processes akin to those used by vertebrates, including image equivalence (Lin \& Chiao, 2017), and contour completion (Zylinski et al., 2012). Cuttlefish also use contextual information from past experiences such as where and when something was learned to modify behavior. Jozet-Alves et al. (2013) demonstrated that cuttlefish use episodic memory to match foraging behavior with food replenishment times. Similarly, Billard et al. (2020) showed source memory via the flexible foraging strategies of cuttlefish. When given crabs (not preferred prey) in the daytime, cuttlefish decided to use either selective or opportunistic foraging on crabs by predicting whether they would be given shrimp during the night (preferred prey). These studies show that cuttlefish store information and use it to make future decisions, because episodic memory and source memory enable the subject to retrieve detailed spatio-temporal representations of past events (Mayes \& Roberts, 2001).

Previous experiments indicate that learning in cuttlefish is dependent upon short-term and long-term memory. These two types of memory differ in molecular mechanisms, as well as the duration over which they are retained. Short-term memory does not involve gene expression, and usually lasts for minutes, while long-term memory lasts hours to a lifetime, requiring new protein synthesis in neural circuits (Izquierdo et 
al., 2002). The cellular mechanisms of short-term and longterm memory in cuttlefish also show some differences. When injected with a protein-synthesis inhibitor, cuttlefish do not show long-term memory of training, but short-term memory is unaffected (Agin et al., 2003). This work suggests that a post-training consolidation period involving protein synthesis is needed to establish long-term memories in cuttlefish. Posttraining protein synthesis is also necessary for long-term memories in vertebrates (Freeman \& Rose, 1999). Bellanger et al. (2003) observed that short-term and long-term memory formation in cuttlefish involved different activities of cholinergic enzymes in the brain, providing additional evidence that different mechanisms control the two memory types.

The inaccessible prey (IP) experiment is a modified and upgraded version of the "prawn in a tube" experiment developed by Sanders and Young (1940). In this procedure, cuttlefish are presented with an inaccessible prey item, such as a shrimp, enclosed in a clear glass or plastic tube. The predatory attack in cuttlefish is visually prompted, and results in the rapid ejection of two specialized feeding tentacles (Messenger, 1968). Cuttlefish strike the tube with feeding tentacles, and, unable to obtain the prey, learn to inhibit their predatory behavior (Messenger, 1971). The number of strikes decreases within trials (acquisition) and across consecutive days of presentation (retention), showing that cuttlefish store information as both short- and long-term memory (Agin et al., 1998; Dickel et al., 2001; Jozet-Alves et al., 2013; Messenger, 1971; Messenger, 1973; Purdy et al., 2006). Long-term retention of the task occurs in adult European cuttlefish, Sepia officinalis (Agin et al., 1998; Cartron et al., 2013; Dickel et al., 1998; Dickel et al., 2001), the pharaoh cuttlefish, Sepia pharaonis (Purdy et al., 2006), and the bobtail squid, Euprymna scolopes, which still retained the task 10 days after the initial training session (Zepeda et al., 2017). While adult cephalopods can acquire and retain the task, juvenile cuttlefish show poor retention in the available literature. Sepia officinalis less than 30 days old show poor 24-h retention, due to immature vertical lobe development (Dickel et al., 2001).

The behavior of S. officinalis may not represent all of Sepia, which contains over 100 species (Jereb \& Roper, 2005). Postembryonic development may be inconsistent for cuttlefish with different habitats and lifespans. Little is known about the ontogeny of long-term memory in other cuttlefish species. In this work, we developed an assay to study memory in the juvenile dwarf cuttlefish, Sepia bandensis, a tropical species native to the Indo-Pacific (Jereb \& Roper, 2005). The dwarf cuttlefish is a suitable model for memory experiments because the species develops quickly, and its relatively small size allows it to be raised and tested in large numbers. However, studies regarding the behavior of this species are lacking.

In behavioral science, there is a need for experimental tools that accurately record subject activity, and simultaneously avoid sensory interference that may impact behavior.
Automated tracking systems quantify many components of subject activity continuously, allowing for comprehensive behavioral analyses and the potential for mathematical modeling of behavior (Sabol et al., 2018). The number of experimental variables measurable through manual observation is limited and may be subjected to bias and observer fatigue (Cullen et al., 2012; Noldus et al., 2001). While manual observation is the only way to record certain behaviors, automated tracking provides an advantage for behaviors involving locomotor or spatial components (Noldus et al., 2001). Automated tracking can consistently quantify multiple aspects of locomotor behaviors, such as distance moved, speed, path tracking, microdynamics of the movement, and orientation of the subject or prey (Noldus et al., 2001). Wireless automated tracking systems have emerged to capture behavior in freely moving subjects, alleviating the disturbance caused by physical tracking devices attached to the animals (Lee et al., 2014). The DanioVision Observation Chamber is a wireless, high-throughput behavioral tracking system that is designed for zebrafish larvae. The closed chamber system offers efficient and comprehensive behavioral monitoring in a controlled environment. Its user-friendly settings can be customized to detect a variety of small animals, such as anemones (Oren et al., 2015), tadpoles (Stanley et al., 2015), and Drosophila larvae (Graham et al., 2016). In this work, we optimized the DanioVision Observation Chamber for use on juvenile dwarf cuttlefish. Attempts at high-throughput tracking are lacking for cephalopods, including cuttlefish. Although adults are too large, the small size of juvenile dwarf cuttlefish permits use of the chamber. Studying juvenile cuttlefish can give insights into adult behavior, as cuttlefish do not experience a larval stage, and hatch as miniature adult forms (Boletzky et al., 2016).

Although learning to stop attacking inaccessible prey via tentacle strike reduction has been well documented in several cuttlefish species, few studies have attempted to understand the microdynamics of the predatory behavioral sequence. Automated tracking systems are valuable for studying predatory behavior in cuttlefish, because the behavior can be divided into three observable phases, which involves orientation and movement of the cuttlefish. The three phases of cuttlefish predatory behavior are attention, positioning, and, finally, the strike (Shinzato et al., 2018; Wells, 1958). The attention phase is visually prompted and begins when the cuttlefish orients its body towards the prey (Shinzato et al., 2018; Wells, 1958). During positioning, the cuttlefish moves towards or away from the prey until it is within striking distance, while the tentacles begin emerging to prepare for the strike (Wells, 1958). During the strike, the prey is seized by terminal suckers on the tentacles, and the prey is rapidly drawn towards the mouth and enveloped by the eight arms (Messenger, 1968). While the predatory response is easily observed, it is not well known how the feedback elicited by failed prey capture affects predatory behavior in its entirety. Repeated failure to capture 
inaccessible prey may also change the cuttlefish's locomotor response and orientation towards the prey; however, these components of the predatory response are difficult to accurately quantify by manual observation.

To our knowledge, only one study has attempted to characterize the effect of inaccessible prey learning on all three phases of the predatory response. Messenger (1971) observed that attention and positioning waned slowly, if at all, relative to striking during a 20-min continuous exposure. From Messenger's initial observation, it can be surmised that strike inhibition is a result of learning and not general fatigue, since only the striking phase is affected. However, Messenger's measurements were based only on the change in frequency of the behaviors for each 5-min interval of the 20-min exposure, and not total duration of attention or distance moved. It would also be useful to know whether the intensity of the behaviors is changed by exposure to inaccessible prey. Moreover, Messenger's study was done using only naïve animals, and the waning of attention and positioning may differ for naïve and previously trained animals reexposed to the procedure. Given this, the development of a more comprehensive method for capturing cuttlefish behaviors is needed to clarify the relationship between the procedure and the whole predatory response.

In this study, our first goal was to determine whether shortterm and long-term memory functioned in 21-day-old dwarf cuttlefish. This is the first study to describe the learning capabilities of $S$. bandensis in any context. We predicted that cuttlefish would display short-term memory of the procedure by reducing strikes during 10-min exposures to inaccessible prey. Similarly, we expected that cuttlefish would display long-term memory by maintaining strike reduction when given a retention test several days after training. By coupling the IP procedure with the DanioVision high-throughput tracking system, our second aim was to determine whether the learning process selectively inhibited the striking phase. We used automated tracking to measure the locomotor components of the predatory response, namely, the duration the body was oriented towards the prey, and the total distance moved by the cuttlefish. We predicted that, in addition to strike reduction, learning would also reduce distance moved by the cuttlefish and reduce the amount of time the front of the animal was oriented towards the prey compared to naïve animals. We expected movement and orientation towards prey to be reduced across training sessions (short-term memory), and reduction of these phases to be maintained during the retention test given several days after training (long-term memory).

\section{Materials and methods}

\section{Animal husbandry}

Sixty S. bandensis eggs were obtained from Blue Zoo Aquatics (Hawthorne, CA, USA). The eggs were wild-caught by the distributor. Eggs were received in two shipments ( 30 eggs per shipment). Eggs were reared in two 20-gal tanks in the Animal Care Facility located within Georgia Southern University. Tanks measured $60 \mathrm{~cm} \times 30.5 \mathrm{~cm} \times 40.5 \mathrm{~cm}$. Tanks were supplied with artificial seawater made with sea salt mix (Instant Ocean). All water used was conditioned with multipurpose dechlorinator (Natural Rapport) before adding it to the tanks. Salinity was maintained at $32-35$ ppt. Water was power filtered (Tetra EX30) using large carbon filter pads (Tetra), and filter pads were changed monthly. Tanks were heated to a temperature of $25 \pm 2{ }^{\circ} \mathrm{C}$ using submersible heaters (Tetra HT30 100-watt). Water changes of 20\% tank volume occurred bi-weekly to control water chemistry. Ammonia, nitrite, and nitrate levels were kept as close as possible to 0 to minimize stress, and $\mathrm{pH}$ was maintained at 8.0-8.2. An overhead light was operated on a 12-h light:dark cycle for both tanks.

Egg masses were kept inside floating fish net breeders (Lee's Aquarium and Pet Products). Net breeders measured $16.5 \times 12.1 \times 13.3 \mathrm{~cm}$. Eggs were continuously aerated by a small air stone mounted beneath the net with a suction cup. Additional aeration of the tanks was provided by a dual air pump (Tetra). At least two air stones per tank were kept running at all times to keep oxygen levels as close as possible to saturation. Eggs were checked daily for hatchlings, and egg casings from hatched animals were removed. Each day, new hatchlings were removed from the egg net and placed in a new net within the tank. Cuttlefish were separated in nets according to age and were housed in groups of four to five. Cuttlefish such as $S$. bandensis form small aggregations, and hatchlings do well in group housing (Fiorito et al., 2015). Cuttlefish were considered 0 days old on the day of hatching. The age of each net was updated daily. Cuttlefish were gently moved to new nets using a ladle and were always kept submerged in water during handling. To minimize stress, cuttlefish were only handled when necessary, and were left undisturbed after daily maintenance and feeding routines were complete. Netting was routinely cleaned and replaced to prevent buildup of algae and other waste materials.

Welfare checks were made at least once a day for all animals. Only animals that showed signs of healthy development (including regular feeding, controlled locomotion, inking, and skin displays) were used for experiments. To our knowledge, no obvious signs of microbial infection (such as skin lesions) were observed for any subjects, including deceased animals. Cuttlefish were not used in experiments until they were 21 days old. Cuttlefish were fed live mysid shrimp daily. Mysids were obtained from Sachs Systems Aquaculture Inc. (St. Augustine, FL, USA).

To prevent further stress on the animals, we did not attempt to determine the sex ratio of the subjects prior to testing. It is difficult to distinguish the sex organ of juvenile males without invasive procedures such as the use of a microscope and 
anesthetics (Christine Bedore and Theresa Gunn, personal comm.).

All studies were conducted in the USA, where there are currently no regulations for the use of cephalopods for experimental purposes. Efforts were made to establish acclimatization, housing, and experimental protocols that adhered to recommended guidelines for the use and care of cephalopods such as those found in Fiorito et al. (2015).

\section{DanioVision tracking system}

Experiments were conducted using a DanioVision Observation Chamber with accompanying EthoVision XT computer tracking software (Noldus Information Technology). The chamber is a closed system equipped with an infrared sensitive camera for recording subjects (Fig. 1a). Hardware settings within the chamber such as white lighting, temperature, and humidity can be modified using EthoVision $\mathrm{XT}$ to create different experimental stimuli. EthoVision XT is also used to create detection settings for identifying and tracking subjects, designing trials, and to collect subject data during experiments. Data collected by the software includes components of subject activity such as distance moved, velocity, and duration of movement.

\section{Experimental plate}

A single experimental plate to be fitted in the DanioVision Observation Chamber was designed for use in all experiments. The plate was created by modifying a six-well culture plate (Corning), so that each well represented an individual cuttlefish arena (Fig. 1b). Each arena measured $9.5 \mathrm{~cm}^{2}$ in area. A clear plastic tube measuring $2 \mathrm{~cm}$ in height and $1.3 \mathrm{~cm}^{2}$ in area was glued into the floor of each arena using clear epoxy. The tube held the inaccessible prey during the procedure. The sixwell culture plate was waterproof, and prevented chemical exchange between the cuttlefish and shrimp. The outside walls of each well were painted opaque with gray acrylic paint to provide isolation for each cuttlefish. The floor remained transparent to allow the arenas to be illuminated by the built-in white light inside the chamber.

\section{Procedures}

\section{Assessment of cuttlefish activity with and without prey}

Prior to designing our learning paradigm, we conducted preliminary tracking trials with the experimental plate to assess differences in cuttlefish activity resulting from the presence or absence of inaccessible prey. These trials occurred with different cuttlefish than those used in the learning paradigm described below (Assessment of short-term memory and longterm memory). Cuttlefish in preliminary trials were 14 days old when trials began. Each trial consisted of three cuttlefish presented with a shrimp in the tube, and three control cuttlefish with tubes filled with mysid tank water only. Trials lasted $20 \mathrm{~min}$. Cuttlefish were tested once a day for 5 days, for a total of 20 trials. Data acquisition followed methods outlined below (Assessment of predatory response phases during learning). Cuttlefish were not fed until trials were completed each day. We observed that cuttlefish presented with inaccessible shrimp moved a greater total distance than control cuttlefish with no shrimp present (see Fig. 3). We attributed this to the cuttlefish's repeated attempts to locate themselves near the prey.

\section{Assessment of short-term memory and long-term memory}

Two replicates of this study were performed. Each replicate contained six trained and six control cuttlefish. Cuttlefish used in the second replicate were from a different shipment (different egg cluster) than cuttlefish used in the first replicate.

When learning experiments began, cuttlefish were not fed until all trials were completed each day. To assess short- and long-term memory in S. bandensis, we designed a learning paradigm based on the IP procedure. The paradigm consisted
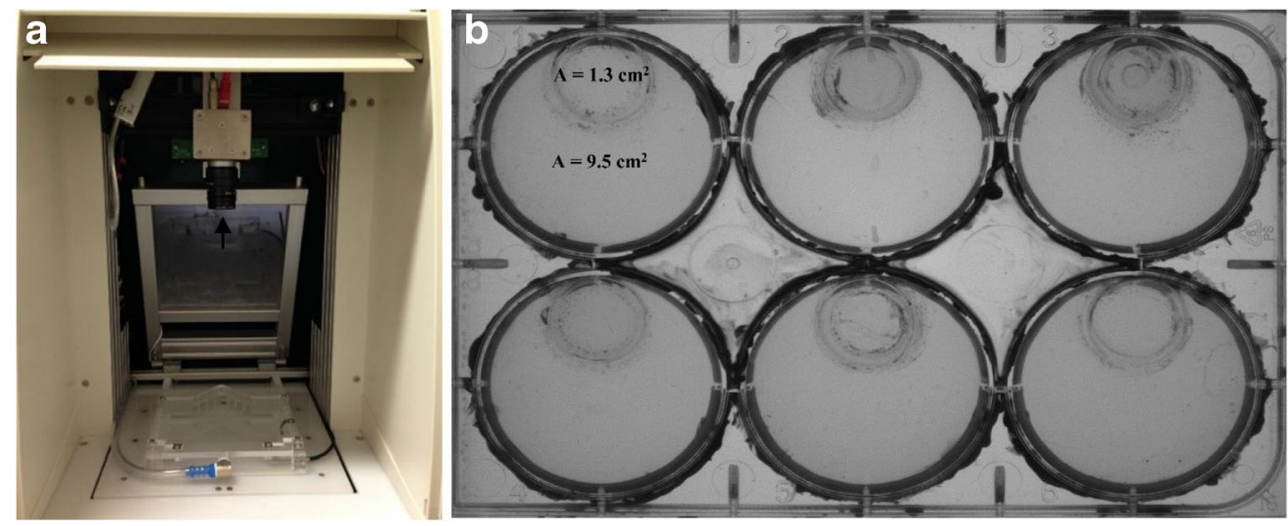

Fig. 1 (a) Photograph of the DanioVision Observation Chamber, with infrared camera (arrow). (b) Photograph of the experimental plate taken from inside the DanioVision Observation Chamber. Tubes for holding mysids were fixed into the top center portion of each arena 
of a training and retention phase. Training and retention phases both began at 10:00 am. During the training phase, 21-day-old cuttlefish underwent five consecutive 10-min trials, with a 20-min resting period between each trial (Fig. 2). Cuttlefish were removed from housing tanks with a cup and placed in a large Tupperware container filled with tank water. The cuttlefish were transported to an adjoining room of the Animal Care Facility, which contained the DanioVision Observation Chamber. Each arena of the experimental plate was filled with $15 \mathrm{~mL}$ of tank water. To begin each trial, cuttlefish were placed individually in each well, and acclimated inside the DanioVision Observation Chamber for $5 \mathrm{~min}$. Each of the shrimp tubes was filled with water from the mysid tank, and a single mysid shrimp was added to each tube using six plastic transfer pipettes. After adding the shrimp, the chamber was closed, and the trial was recorded using EthoVision XT. The built-in white light operated at $15 \%$ intensity during trials to ensure prey visibility.

Cuttlefish were never left in the plate longer than the 10min trial period and were closely monitored throughout all trial recordings. We did not observe any prolonged stress from subjects within the artificial environment, and all test subjects tested exhibited predatory behavior by watching prey and striking the tube at least once during testing. After trials were complete, cuttlefish were immediately returned to the housing tanks and were fed mysids. Cuttlefish quickly resumed normal tank behavior and readily captured freely swimming prey after the trials. No visible injuries were sustained by the subjects, and no animals died during experimental recordings. All subjects survived the full duration of the experiment.

To assess short-term memory acquired within trials, each trial was divided into two continuous 5-min intervals, and the number of strikes for the first and last 5 min were compared (Fig. 2). Total strikes were obtained by combining the strikes for both intervals for each individual (12 cuttlefish). To assess learning between trials, strikes made during the last training session were compared to strikes made during the initial training session.

Four days after the training day, the same cuttlefish underwent a single 10-min retention test to assess long-term memory of the inaccessible prey procedure (Fig. 2). For control, we performed the same test on naïve cuttlefish on the retention day. The naïve cuttlefish were the same age as the trained cuttlefish (21 days).

\section{Assessment of predatory response phases during learning}

Since our preliminary experiments revealed a clear effect of prey presence on cuttlefish activity, we aimed to determine whether the three predatory response phases (attention, positioning, and striking) would change as a result of learning the inaccessible prey paradigm we designed for 21-day-old cuttlefish. To accomplish this, we tracked orientation and total movement of the cuttlefish using EthoVision XT software. Subject tracking occurred from saved video files after the experiment was recorded in its entirety. EthoVision XT uses center-point tracking to record total movement of each individual within the arena (Fig. $3 a)$. The position of the center point is compared across consecutive frames to generate the movement path of the cuttlefish (Fig. $3 b)$. In addition to center-point tracking, we used another tracking method called "head-to-tail" tracking. Head-to-tail tracking places a tracking point on the head and tail of the animal, such that orientation and movement of the whole body within the arena can be analyzed (Fig. 3a and b). Separate detection settings were used to track the movement of each mysid shrimp (Fig. 3c).

Size calibration of the experimental plate was set at 12.5 $\mathrm{cm}$ (length of the plate). Subject tracking occurred at a rate of 30 frames/s. Subject tracking occurred via the static subtraction method, in which changes in each frame are compared to a static background reference image, in this case, the image of the empty experimental plate.

\section{Attention}

To assess the attention phase, we measured the amount of time the cuttlefish was oriented towards the prey. EthoVision XT was used to divide each cuttlefish arena into two zones. The arenas were divided in half, with the top zone containing the shrimp tube (Fig. 3d). We used "head-to-tail" tracking of the cuttlefish to record their time spent oriented towards prey. Orientation towards prey was measured by the total time the front of the cuttlefish was oriented towards the zone containing the shrimp tube (Fig. 3d). We used total duration of orientation towards the tube to estimate attention, because the cuttlefish often remained facing the tube after completing a strike. Unless the cuttlefish turn away from the tube after each tentacle strike, it is difficult to tell exactly when the next attention phase begins. Moreover, the cuttlefish do not always

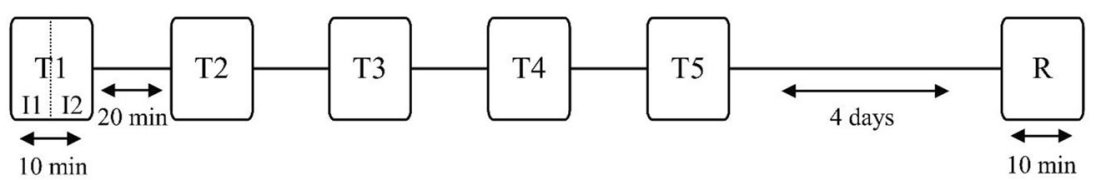

Fig. 2 Schematic of timeline of the inaccessible prey (IP) learning paradigm. On the first day, cuttlefish were exposed to inaccessible prey for five consecutive training sessions (T1-T5). Training sessions lasted 10 min each with a 20-min resting period between sessions. Shown by the dotted line in the first training session, $\mathrm{T} 1$, sessions were divided into two continuous, 5-min intervals (I1 and I2) to assess changes in the predatory response within sessions (short-term memory). Four days after training, a single, 10-min exposure to inaccessible prey was given as a retention test $(\mathrm{R})$ to assess long-term memory 


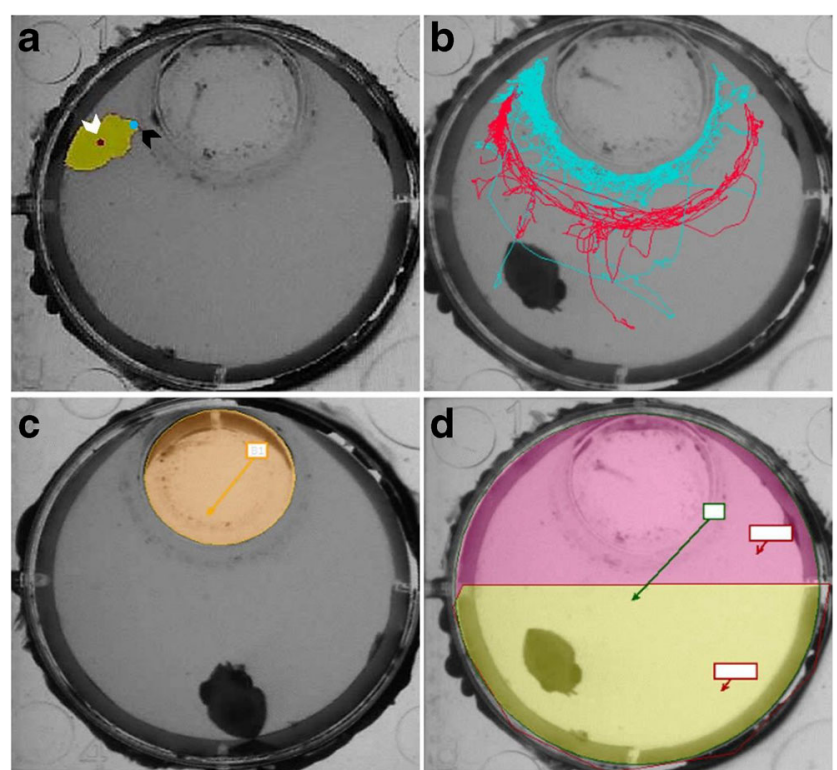

Fig. 3 Experimental setup designed with EthoVisionXT. (a) Detection of cuttlefish inside the experimental plate. The red center point marker appeared on the dorsoanterior portion of the mantle (white arrowhead). The head point appeared on the tip of the arm crown (black arrowhead). Movement of the center point is used to generate subject data such as distance moved, velocity, frequency of rotations, and movement paths. (b) Tracking acquired from cuttlefish. Distance moved is shown by the red line. Orientation of the front of the cuttlefish is shown by the blue line. Mysid shrimp were present simultaneously within the arena but did not interfere with cuttlefish tracking. (c) Arena settings for detecting mysid shrimp. The orange shading indicates that arena boundaries were confined to the inside of each tube. (d) Arena settings for cuttlefish. Each arena was divided into two zones. The top zone (pink) contained the shrimp tube; the bottom zone (yellow) was empty

immediately begin moving after seeing the prey, so even if the cuttlefish appears inactive, it may still be watching the prey. We predicted the amount of time spent facing the shrimp zone would decrease across sessions as a result of learning.

\section{Positioning}

Total distance moved by each cuttlefish was recorded from the center-point tracker to measure the amount of positioning. (Fig. 3a and d). We used total movement to estimate positioning, because movement towards or away from the prey did not always result in a full tentacle strike (see below, Strike). Unless the cuttlefish always strikes after positioning itself, it becomes difficult to separate positioning from general locomotion, especially if the cuttlefish continues facing the prey. The total distance moved by each mysid was also measured to assess any differences in prey activity (Fig. 3c).

\section{Strike}

After all trials had been recorded, the number of tentacle strikes by each cuttlefish was manually recorded with a tally counter. While automated tracking is more advantageous for measuring locomotor behaviors such as total movement and body orientation, the processes underlying the tentacle strike itself are too variable to be reliably recorded with an automated system. The tentacle strike begins when tentacles are extended beyond the body of the cuttlefish. However, the cuttlefish often extended feeding tentacles to touch the tube without completing the ejection. This behavior was not counted as a strike. While reaching out to touch the tube with tentacles may show intent to capture prey, it is ambiguous for an assessment of actual strikes, because an exposed tentacle does not always culminate in a full predatory strike, even when no barrier is present between the cuttlefish and the prey (personal observations). It is known that striking behavior can vary among individuals and may not be as reflexive as originally thought (Zoratto et al., 2018). We often observed cuttlefish slowly retracting visible tentacles and waiting until the prey was within the preferred striking distance, which tends to be about one mantle length (Hanlon \& Messenger, 1996; Messenger, 1968). In other cases, once the tentacle was retracted, the cuttlefish decided not to strike at all. Strikes were counted for instances in which the tentacles were visibly extended towards the tube, followed by a seizure attempt involving rapid retraction of the tentacles and spreading of the arms in anticipation of drawing prey towards the mouth.

\section{Statistics}

Data generated by EthoVision XT were exported as Microsoft Excel files. All statistical analyses were conducted in JMP 13.1.0 (SAS Institute, Inc., Cary, NC, USA). Nonparametric analyses (Wilcoxon test, Kruskal Wallis test, and Sign test) were used when data failed to meet the assumptions for parametric tests (normally distributed data and equal variance). The Shapiro-Wilk test was used to assess normalcy. Levene's test was used to assess equality of variances. Strikes were compared for intervals and trials by pooling the data for all subjects. The pooling of strikes has been used to assess learning in similar studies (Agin et al., 1998; Dickel et al., 1998; Dickel et al., 2001). Similarly, orientation and movement data were pooled across subjects and trials for analysis.

\section{Results}

\section{Cuttlefish activity with and without prey}

The presence of inaccessible prey had a visible effect on the movement patterns of cuttlefish (Fig. 4). Our preliminary data demonstrated that cuttlefish increased the amount of time spent moving when a shrimp was present (Wilcoxon test; $\chi^{2}$ $=16.8 ; \mathrm{p}<0.0001)$. Cuttlefish also increased total distance moved when a shrimp was present (Wilcoxon test; $\chi^{2}=9.29$; $\mathrm{p}=0.002$ ). This provided evidence that movement duration and distance moved can be used to measure prey interest, 


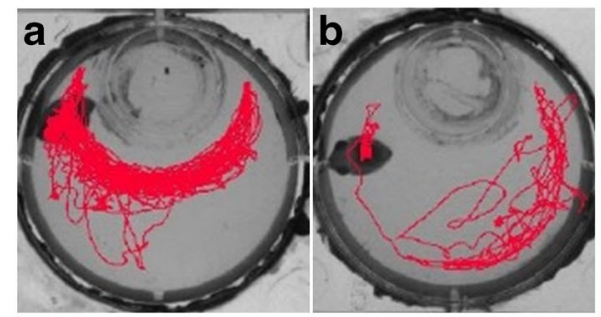

C

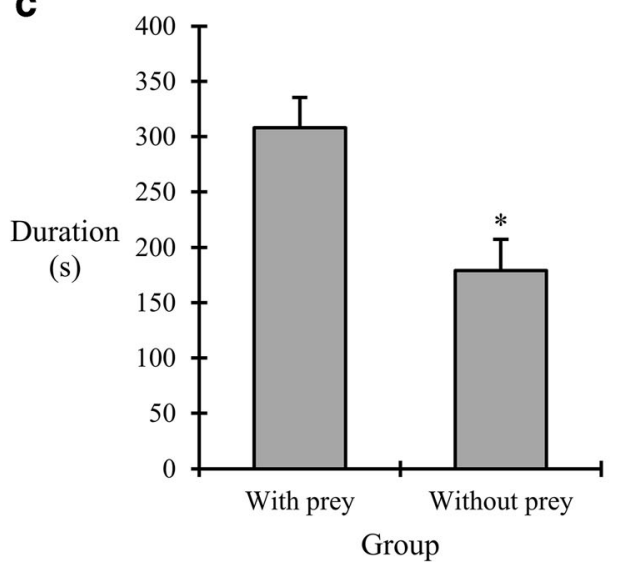

Fig. 4 Preliminary cuttlefish tracking data collected from a total of 20 trials lasting 20 min each. (a) Movement path of cuttlefish with shrimp present. (b) Movement path of cuttlefish without shrimp. (c) Movement duration of cuttlefish presented with prey and cuttlefish without prey. (d)

since both duration and total movement were significantly influenced by the presence of prey. Given this preliminary data, we asked whether EthoVision XT tracking could be used to quantify changes in cuttlefish activity related to learning.

\section{Assessment of short-term memory}

To determine whether juvenile $S$. bandensis exhibited short-term memory by assessing the number of strikes

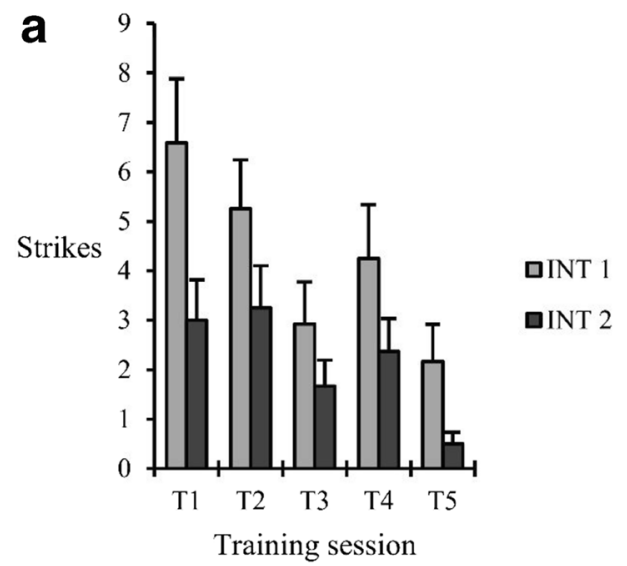

Fig. 5 (a) Strikes made during Interval 1 and Interval 2 for each training session. Trials were divided into two continuous, 5-min intervals and the number of strikes per interval were counted (INT $1=$ Interval 1 ; INT $2=$ Interval 2). Data are presented as mean total strikes for each interval + d

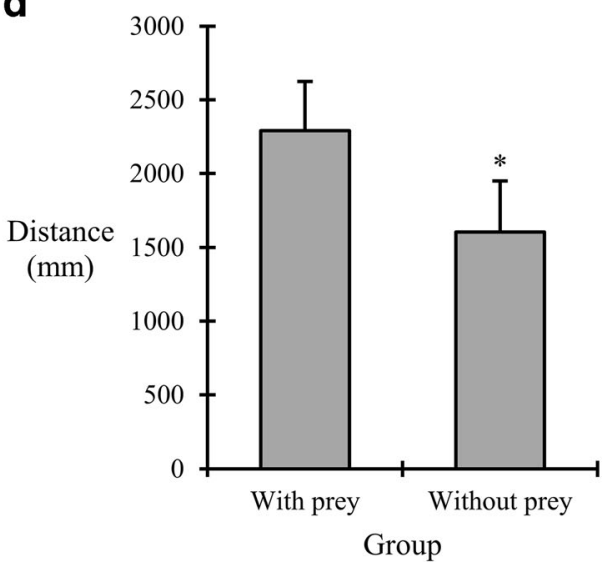

Total movement of cuttlefish presented and cuttlefish without prey. Data presented are mean + SEM $(n=24)$. Asterisks represent significant differences for cuttlefish without prey

made during Interval 1 and Interval 2, strikes made during each interval were counted and counts for each training session were pooled for data analysis. Strikes during Interval 1 differed from Interval 2 (Fig. 5a, Sign test; $M=14.0, p<0.0001)$. To determine whether learning occurred across training, strikes made during intervals of $\mathrm{T} 5$ were compared to intervals of $\mathrm{T} 1$. Strikes made during Interval 1 decreased from T1, the first training session, to $\mathrm{T} 5$, the last training session

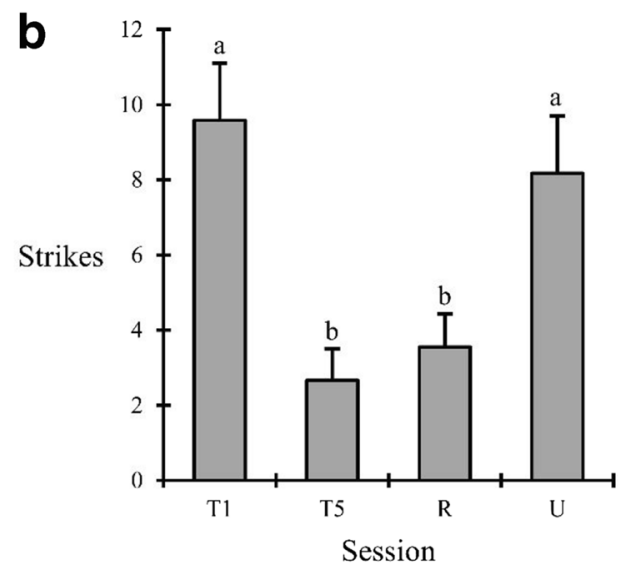

$\operatorname{SEM}(\mathrm{n}=12)$. $(\mathbf{b})$ Comparison of total strikes for T1, T5, retention $(\mathrm{R} ; \mathrm{n}=$ 12) and untrained cuttlefish (U; $n=12)$. Data are presented as mean total strikes + SEM. Different letters represent significance at $\alpha=0.05$ using Steel Dwass multiple comparisons 
(Fig. 5a, Wilcoxon test; $\chi^{2}=6.29, \mathrm{p}=0.01$ ). Strikes made during Interval 2 also decreased from T1 to T5 (Fig. 5a, Wilcoxon test; $\chi^{2}=6.57, \mathrm{p}=0.01$ ).

\section{Assessment of long-term memory}

To assess establishment of long-term memory, cuttlefish underwent a retention test 4 days after training. The same test was conducted on naïve cuttlefish. Total strikes differed among sessions (Fig. 5b, Kruskal Wallis test; $\chi^{2}=12.9, \mathrm{p}=$ 0.002). There were less strikes during Retention than T1 (Fig. $5 b$, Steel Dwass multiple comparisons; $Z=2.84 p=0.02$ ), but Retention and T5 did not differ (Fig. 5b, $Z=-0.75, \mathrm{p}=0.87$ ). Total strikes for untrained cuttlefish were greater than total strikes for T5 (Fig. 5b, $Z=2.35, p=0.039$ ), but strikes for untrained cuttlefish did not differ from $\mathrm{T} 1(\mathrm{Z}=-0.49, \mathrm{p}=$ $0.96)$.

\section{Assessment of attention}

We measured attention as the amount of time spent oriented towards prey by dividing each arena into two zones and placing a tracking point on the tip of the arm crown to determine the orientation of the cuttlefish. Cuttlefish spent more time oriented towards the shrimp zone than the empty zone (Fig. 6a, Sign test; $\mathrm{M}=-15.5, \mathrm{p}<0.0001$ ). Duration oriented towards the shrimp zone did not differ across sessions (Fig. 6a, Kruskal Wallis test; $\chi^{2}=2.7, p=0.44$ ), nor did duration oriented towards the empty zone (Fig. 6a, Kruskal Wallis test; $\left.\chi^{2}=1.65, \mathrm{p}=0.65\right)$.

\section{Assessment of positioning}

We measured the amount of positioning by tracking total movement of the cuttlefish. We aimed to determine whether the total movement would change as a result of learning. Total movement did not differ among sessions (Fig. 6b, Kruskal Wallis test; $\chi^{2}=7.66, p=0.05$ ). Total movement by mysids

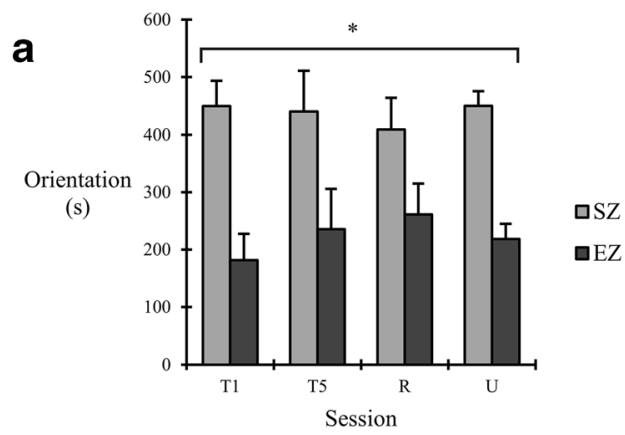

Fig. 6 (a) Total time cuttlefish spent oriented towards the shrimp zone and the empty zone (SZ = shrimp zone; $\mathrm{EZ}=$ empty zone). Data are presented as mean duration per session + SEM $(n=24)$. Asterisk represents significant difference between $\mathrm{SZ}$ and $\mathrm{EZ}$ duration for data also did not differ among sessions (Fig. 6b, Wilcoxon test; $\chi^{2}$ $=1.9, p=0.58)$, so we do not expect that prey movement had an effect on the amount cuttlefish movement.

\section{Discussion}

Our results show that short-term memory functions in 21-dayold $S$. bandensis, as the number of strikes against inaccessible prey was significantly greater for Interval 1 than Interval 2 (Fig. 5a). This is consistent with findings using $S$ officinalis, for which short-term memory operates as early as 8 days old (Agin et al., 1998; Dickel et al., 1998). The reduction in strikes between $\mathrm{T} 1$ and $\mathrm{T} 5$ demonstrated that learning also occurred between trials (Fig. 5a). Messenger (1971) showed that establishment of memory in cuttlefish is biphasic, with short-term memory decaying after $20 \mathrm{~min}$, and long-term memory appearing after $1 \mathrm{~h}$. The biphasic memory curve has also been described for the chambered nautilus (Nautilus pompilius), a shelled cephalopod (Crook \& Basil, 2008). The reduction in strikes between $\mathrm{T} 1$ and $\mathrm{T} 5$ of training may represent the activation of long-term memory stores in S. bandensis, as approximately $2 \mathrm{~h}$ elapsed between $\mathrm{T} 1$ and T5. The 20-min resting interval may have been too long for short-term memory to be maintained between each trial.

S. officinalis does not exhibit 24-h retention until 30 days of age (Dickel et al., 2001). Surprisingly, we found evidence that 21-day-old $S$. bandensis retains memory of the IP procedure for up to 4 days (Fig. 5b). The strikes of trained cuttlefish during the retention test were reduced compared to $\mathrm{T} 1$ of training (Fig. 5b), but were similar to T5 (Fig. 5b). This suggests that the cuttlefish did not revert to their original response rate, despite the fact that 4 days elapsed between training and the retention test. Concomitant with this, the response of untrained cuttlefish was similar to T1 (Fig. 5b) but differed from T5 (Fig. 5b).

Retention improves with increased vertical lobe development, the center for memory processing in cephalopods (Dickel et al., 2001; Mather \& Kuba, 2013). While retention

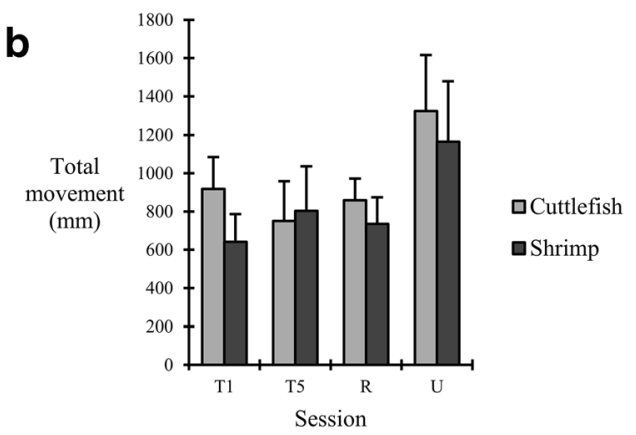

pooled across sessions $(\mathrm{p}<0.0001)$. (b) Total movement of cuttlefish $(n=24)$ and shrimp $(n=42)$ per testing session. Data are presented as mean total movement + SEM 
does not emerge in S. officinalis until 30 days (Dickel et al., 2001), the ontogeny of long-term memory-related behaviors in juveniles has not been described in other members of Sepia. Moreover, environmental factors related to the ontogeny of memory have not been fully examined within juvenile Sepia. Maturity of the vertical lobe may show interspecific variation because cephalopod development depends upon multiple environmental factors, including temperature (Boletzky, 1994; Domingues et al., 2001; Forsythe \& Hanlon, 1988) and environmental complexity (Yasumoro and Ikeda, 2016, 2018).

Cephalopod development is accelerated with increasing temperature (Boletzky, 1994). Forsythe and Hanlon (1988) found that a rearing temperature increase of $5^{\circ} \mathrm{C}$ sped the development of Octopus bimaculoides eggs, accelerated sexual maturity, and reduced lifespan. Similarly, Domingues (2001) observed that $S$. officinalis hatchlings cultured at the upper limit of their geographic temperature range had a higher growth rate than cuttlefish raised at lower temperatures. Considering its effect on lifespan and reproductive behavior, environmental temperature could influence the development of the brain and other organs. Tropical species reared in warmer water may show accelerated development of neural structures related to learning, compared to temperate species raised at cooler temperatures. This could lead to plasticity in the onset of observable behaviors commonly used for learning assays, such as IP strike inhibition. However, the effect of rearing temperature on the ontogeny of learning and memory is not well known in cuttlefish.

Other studies have shown that ontogeny of learning and other behaviors is affected by environmental enrichment (Yasumoro \& Ikeda 2016, 2018). S. pharaonis raised in enriched tank settings resembling complex environments (natural substrate, objects, conspecifics) show early maturity of cryptic coloration behaviors not seen in cuttlefish raised in deprived tanks (Yasumoro \& Ikeda, 2016). Interestingly, Yasumoro and Ikeda (2018) showed enrichment affects ontogeny of IP learning in S. pharaonis. Individuals kept in enriched environments were able to continue learning with increasing age, while individuals in poor environments lost the ability to learn with age. However, the cuttlefish were not tested at ages earlier than 74 days. The effect of environmental richness on the ontogeny of memory in younger cuttlefish (30 days or less) remains to be explored.

These environmental factors offer a potential explanation to our observation that $S$. bandensis show retention of the IP procedure at 21 days old. S. bandensis and S. officinalis occupy two distinct habitats, with varying temperature and visual complexity. S. bandensis is native to warm tropical reefs of the Indo-Pacific, while $S$. officinalis resides in temperate waters of the east Atlantic, as well as in the North, Baltic, and Mediterranean seas (Jereb \& Roper, 2005). Tropical waters are clear and may be more visually complex than the turbid waters that S. officinalis inhabit (Yasumoro \& Ikeda, 2018). In visually complicated environments, there may be need for early development of neural structures responsible for visual learning. This would provide an advantage to juvenile cuttlefish, which receive no parental care, and must survive independently after hatching (Yasumoro \& Ikeda, 2018).

Relationships between habitat complexity and neural development are shown in vertebrate taxa, such as teleosts (Axelrod et al., 2018; Kotrschal et al., 1998). Fish that inhabit clear tropical waters tend to show enhanced neural growth of visual centers, whereas in turbid environments, visual importance is reduced and chemosensory structures are enhanced (Kotrschal et al., 1998). Recently, Axelrod et al. (2018) showed that pumpkinseed sunfish living in complex littoral environments had larger brains than the same sunfish living in pelagic environments. With this evidence, the relationship between habitat and cuttlefish brain development needs further consideration.

Within Sepia, developmental stages have only been documented for S. officinalis (Boletzky et al., 2016), and $S$. pharaonis (Lee et al., 2016). The eyes develop faster in the tropical S. pharaonis than in S. officinalis (Lee et al., 2016), but whether this is related to habitat is unknown. Given that the vertical lobe is used for visual learning and memory (Boycott \& Young, 1957), it is interesting to consider whether the vertical lobe matures faster in tropical cuttlefish species that show rapid eye development. Kobayashi et al. (2013) showed that growth of the vertical lobe is rapid compared to motor regions in young oval squid, another tropical species. Enhanced development of visual learning centers could also explain earlier retention of the IP paradigm in tropical $S$. bandensis because the experiment involves visual cues in the form of live prey. In addition to live prey, detection of the transparent prey tube via polarized vision could convey cues related to the learned inhibition of strikes (Cartron et al., 2013). More work detailing the early development of $S$. bandensis is needed to understand differences in the ontogeny of learning and memory in this species.

Since Messenger's 1971 study, the effect of the IP procedure on all three phases of the predatory response has not been addressed. Messenger found that, relative to striking, attention and positioning waned slowly, if at all, in naïve cuttlefish given a continuous 20-min exposure to enclosed prey. However, the assessments of attention and positioning were limited to what Messenger called a "very crude measure" relative to the measure of strikes. While Messenger measured the additional two phases within trials, it is unknown whether the procedure affects the phases for different training regimes, such as successive trials. Since it is known that the level of striking is affected by repeated sessions, it would also be useful to know whether repetition or re-exposure causes the other two phases to wane in a previously trained animal compared to a naïve one.

The advent of automated tracking systems since Messenger's work has allowed for more comprehensive and 
accurate analyses of complex behaviors, but these methods have yet to be fully applied to cuttlefish and other cephalopods. Our analysis of predatory behavior with DanioVision showed that neither total movement nor the orientation of the cuttlefish towards inaccessible prey changed as a result of learning. This further suggests that the IP procedure selectively inhibits the strike, and not the entire predatory sequence. Cuttlefish spent the same amount of time oriented towards prey across trials (Fig. 6a). Across trials, trained cuttlefish remained active around the shrimp tubes, despite the fact that they struck less with tentacles (Fig. 6b). Our analysis provides evidence that the reduction of strikes is a real learning effect, and not a result of fatigue gained across repeated sessions. If cuttlefish stopped striking due to fatigue, it should be expected that orientation towards prey and total movement also decline across sessions. Instead, we found that cuttlefish inhibited the striking phase, but similar changes were not reflected in the attention and positioning phases.

Selective inhibition of the striking phase may result from sensory cues associated with the tentacle strike. Messenger (1971) also posited that learning occurred via strike-contingent pain from tentacles hitting the tube. He observed that cuttlefish with their tentacles cut could still learn to stop attacking, but that it took longer than cuttlefish with tentacles. The suckers on the terminal ends of tentacles contain many tactile receptors and chemoreceptors (Graziadei, 1964; Wells, 1964). Touch receptors in the suckers may convey a pain signal that the cuttlefish learns to associate with striking against the tube. Inhibition of predatory response phases (attention, positioning, striking) may only occur when a negative stimulus (such as pain) is associated with the specific response phase. Cuttlefish readily captured a freely swimming prey item immediately after conclusion of our procedure, and also in Dickel et al. (1998, 2001), which is consistent with our finding that interest in the prey is not inhibited by the procedure or hindered by fatigue. Purdy et al. (2006) observed that IP learning was stimulus-specific in S. pharaonis, indicating that inhibition of striking may result from associative learning rather than fatigue. After learning to inhibit attacks against inaccessible fish, cuttlefish reverted to high striking rates when the prey was switched to inaccessible shrimp. In contrast to this, Carton et al. (2013) did not observe a stimulusspecificity effect on IP learning, finding that learned inhibition by $S$. officinalis was not affected when shrimp were replaced by crabs.

If cuttlefish learn via associative processes during the IP procedure, it is necessary to examine additional cues related to predatory behavior. Recent work has shown that multiple sensory inputs from the prey tube could provide context relevant to IP learning. Carton et al. (2013) suggested that cuttlefish see the prey tube and recognize it as an obstacle preventing access to the prey. Cuttlefish may also detect tactile cues from the tube by gripping it with the arms during the procedure (Dickel et al., 2013). We observed that, after striking with the tentacles, cuttlefish would often grip the tube with the arms for long periods, supporting the idea that tactile cues from the tube could be associated with learned strike inhibition. Cues from the prey tube may inhibit striking but could be insufficient to reduce the overall interest in the prey. This is because prey presence is a strong visual stimulus for initiating attention and positioning phases in cuttlefish (Shinzato et al., 2018).The ability to perceive the tube as an obstacle to successful prey capture could explain why cuttlefish stop striking, but otherwise remain attentive towards prey, and quickly capture free prey after the procedure. More work is necessary to understand the multisensory inputs related to IP learning, as well the dynamics of the predatory response phases during the procedure.

\section{Conclusion}

The DanioVision system allowed for a comprehensive evaluation of the multi-phased cuttlefish predatory response towards inaccessible prey. We found that, when presented with inaccessible prey, cuttlefish selectively inhibit tentacle strikes without reducing the amount of time oriented towards prey, or total distance moved. We present DanioVision as a powerful tool for analyzing dynamic behaviors in cuttlefish. Juvenile $S$. bandensis are an excellent model for the DanioVision system due to their small size, and their capacity to exhibit many adult behaviors early in development. We encourage further use of similar automated tracking methods, as they could lend renewed understanding of cuttlefish behavior for a wide array of experimental behavioral assays.

Acknowledgements We would like to acknowledge Theresa Gunn, Dr. Christine Bedore, and Bret Grasse for their valuable advice on cuttlefish rearing and handling.

Funding This work was supported by the College of Science and Mathematics, Georgia Southern University, Statesboro, GA, USA; the Graduate Student Organization, Georgia Southern University, Statesboro, GA, USA; and the University Faculty Research Funds, Georgia Southern University, Statesboro, GA, USA.

\section{Compliance with ethical standards}

Conflict of interest none

\section{References}

Agin, V., Dickel, L., Chichery, R., Chichery, M.P., 1998. Evidence for a specific short-term memory in the cuttlefish, Sepia, Behavioural Processes 43, 329-34. 
Agin, V., Chichery, R., Maubert, E., Chichery, M.P., 2003. Time-dependent effects of cycloheximide on long-term memory in the cuttlefish. Pharmacology, Biochemistry, and Behavior 75, 141-146.

Alves, C., Chichery, R., Boal, J.G., Dickel, L., 2007. Orientation in the cuttlefish Sepia officinalis: Response versus place learning. Animal Cognition 10, 29-36.

Axelrod, C.J., Laberge, F., Robinson, B.W., 2018. Intraspecific brain size variation between coexisting sunfish ecotypes. Proceedings of the Royal Society B: Biological Sciences 285.

Bellanger, C., Dauphin, F., Chichery, M.P., Chichery, R., 2003. Changes in cholinergic enzyme activities in the cuttlefish brain during memory formation. Physiology \& Behavior 79, 749-756.

Billard, P., Schnell, A.K., Clayton, N.S., Jozet-Alves, C., 2020. Cuttlefish show flexible and future-dependent foraging cognition. Biology Letters $16,1-5$.

Boletzky, S. v., 1994. Embryonic development of cephalopods at low temperatures. Antarctic Science 6, 139-142.

Boletzky, S. V., Andouche, A., Aud-Ponticelli, L.B., 2016. A developmental table of embryogenesis in Sepia officinalis. Vie Milieu 66, $11-23$.

Boycott, B.B., 1961. The Functional Organization of the Brain of the Cuttlefish Sepia officinalis. Proceedings of the Royal Society of London 153, 503-534.

Boycott, B.B., Young, J.Z., 1957. Effects of interference with the vertical lobe on visual discriminations in Octopus vulgaris Lamarck. Proceedings of the Royal Society B: Biological Sciences 146, 439-459.

Cartron, L., Darmaillacq, A.S., Dickel, L., 2013. The "prawn-in-the-tube" procedure: What do cuttlefish learn and memorize? Behavioural Brain Research 240, 29-32.

Crook, R., Basil, J., 2008. A biphasic memory curve in the chambered nautilus, Nautilus pompilius L. (Cephalopoda : Nautiloidea). The Journal of Experimental Biology 211, 1992-1998.

Cullen, D.A., Sword, G.A., Simpson, S.J., 2012. Optimizing multivariate behavioural syndrome models in locusts using automated video tracking. Animal Behaviour 84, 771-784.

Dickel, L., Chichery, M.P., Chichery, R., 1998. Time differences in the emergence of short- and long-term memory during post-embryonic development in the cuttlefish, Sepia, Behavioural Processes 44, 81 6.

Dickel, L., Chichery, M.P., Chichery, R., 2001. Increase of learning abilities and maturation of the vertical lobe complex during postembryonic development in the cuttlefish, Sepia, Developmental Psychobiology 39, 92-98.

Dickel, L., Darmaillacq, A.S., Jozet-Alves, C., Bellanger, C., 2013. Learning, Memory, and Brain Plasticity in Cuttlefish (Sepia officinalis), in: Handbook of Behavioral Neuroscience. pp. 318 333

Domingues, P.M., Kingston, T., Sykes, A., Andrade, J.P., 2001. Growth of young cuttlefish, Sepia officinalis (Linnaeus 1758) at the upper end of the biological distribution temperature range. Aquaculture Research 32, 923-930.

Fiorito, G., Affuso, A., Basil, J., Cole, A., Girolamo, P. D., D’Angelo, L., ... Andrews, P. L., 2015. Guidelines for the Care and Welfare of Cephalopods in Research -A consensus based on an initiative by CephRes, FELASA and the Boyd Group. Laboratory Animals, 4, 190.

Forsythe, J.W., Hanlon, R.T., 1988. Effect of temperature on laboratory growth, reproduction and life span of Octopus bimaculoides. Marine Biology 379, 369-379.

Freeman, F.M., Rose, S.P.R., 1999. Expression of Fos and Jun proteins following passive avoidance training in the day-old chick. Learning \& Memory 6, 389-397.

Graham, S., Rogers, R.P., Alper, R.H., 2016. An automated method to assay locomotor activity in third instar Drosophila melanogaster larvae. Journal of Pharmacological and Toxicological Methods, 77, 76-80.

Grasso, F.W., Basil, J.A., 2009. The evolution of flexible behavioral repertoires in cephalopod molluscs. Brain, Behavior and Evolution 74, 231-245.

Graziadei, P., 1964. Receptors in the Sucker of the Cuttlefish. Nature 203, 384-386.

Hanlon, R., Messenger, J., 1996. Cephalopod Behaviour. Cambridge University Press.

Izquierdo, L.A., Barros, D.M., Vianna, M.R.M., Coitinho, A., De Silva, T.D., Choi, H., Moletta, B., Medina, J.H., Izquierdo, I., 2002. Molecular pharmacological dissection of short- and long-term memory. Cellular and Molecular Neurobiology 22, 269-287.

Jereb, P., Roper, C., 2005. Cephalopods of the world. An annotated and illustrated catalogue of cephalopod species known to date. Volume 1. Chambered nautiluses and sepioids (Nautilidae, Sepiidae, Sepiolidae, Sepiadariidae, Idiosepiidae, and Spirulidae). FAO Species Catalogue for Fishery Purposes.

Jozet-Alves, C., Bertin, M., Clayton, N.S., 2013. Evidence of episodiclike memory in cuttlefish. Current Biology 23, 1033-1035.

Kobayashi, S., Takayama, C., Ikeda, Y., 2013. Ontogeny of the brain in oval squid Sepioteuthis lessoniana (Cephalopoda: Loliginidae) during the post-hatching phase. Journal of the Marine Biological Association of the United Kingdom 93, 1663-1671.

Kotrschal, K., van Staaden, M.J., Huber, R., 1998. Fish brains: evolution and environmental relationships. Reviews in Fish Biology and Fisheries 8, 373-408.

Lee, B., Kiani, M., Ghovanloo, M., 2014. A smart homecage system with 3D tracking for long-term behavioral experiments. 2014 36th Annu. Int. Conf. IEEE Eng. Med. Biol. Soc.

Lee, M., Lin, C., Chiao, C., Lu, C., 2016. Reproductive Behavior and Embryonic Development of the Pharaoh Cuttlefish, Sepia pharaonis (Cephalopoda : Sepiidae). Zoological Studies 41, 1-16.

Lin, I.R., Chiao, C.C., 2017. Visual equivalence and amodal completion in cuttlefish. Frontiers in Physiology 8, 1-14.

Manns, J.R., Eichenbaum, H., 2009. A cognitive map for object memory in the hippocampus. Learning \& Memory 16, 616-624.

Mather, J.A., Kuba, M.J., 2013. The cephalopod specialties: complex nervous system, learning, and cognition. Canadian Journal of Zoology 91, 431-449.

Mayes, A.R., Roberts, N., 2001. Theories of episodic memory. Philosophical Transactions of the Royal Society B: Biological Science 356, 1395-1408.

Messenger, J.B., 1968. The visual attack of the cuttlefish Sepia officinalis. Animal Behaviour 16, 342-357.

Messenger, J.B., 1971. Two stage recovery of a response in Sepia. Nature 232, 202-203.

Messenger JB. 1973. Learning in the cuttlefish, Sepia, Animal Behaviour $21,801-826$

Noldus, L.P.J.J., Spink, A.J., Tegelenbosch, R.A.J., 2001. EthoVision : A versatile video tracking system for automation of behavioral experiments. Behav. Res. Methods, Instruments, Comput. 33, 398-414.

Oren, M., Tarrant, A.M., Alon, S., Simon-Blecher, N., Elbaz, I., Appelbaum, L., Levy, O, 2015. Profiling molecular and behavioral circadian rhythms in the non-symbiotic sea anemone Nematostella vectensis. Scientific Reports 5, 114-118.

Purdy, J.E., Roberts, A C., Garcia, C. A, 1999. Sign tracking in cuttlefish (Sepia officinalis). Journal of Comparative Psychology 113, 443 449.

Purdy, J.E., Dixon, D., Estrada, A., Peters, A., Riedlinger, E., Suarez, R., 2006. Prawn-in-a-tube procedure: Habituation or associative learning in cuttlefish? The Journal of General Psychology 133, 131-152.

Sabol, A.C., Solomon, N.G., Dantzer, B., 2018. How to Study Socially Monogamous Behavior in Secretive Animals ? Using Social Network Analyses and Automated Tracking Systems to Study the 
Social Behavior of Prairie Voles. Frontiers in Ecology and Evolution 6, 1-13.

Sanders, F.K., Young, J.Z., 1940. Learning and other functions of the higher nervous centres of Sepia. Journal of Neurophysiology 3, 501526.

Scatà, G., Jozet-Alves, C., Thomasse, C., Josef, N., Shashar, N., 2016. Spatial learning in the cuttlefish Sepia officinalis: Preference for vertical over horizontal information. The Journal of Experimental Biology 219, 2928-2933.

Shinzato, S., Yasumuro, H., Ikeda, Y., 2018. Visual Stimuli for the Induction of Hunting Behavior in Cuttlefish Sepia pharaonis. The Biological Bulletin 234, 106-115.

Stanley, J.K., Lotufo, G.R., Biedenbach, J.M., Chappell, P., Gust, K.A., 2015. Toxicity of the conventional energetics TNT and RDX relative to new insensitive munitions constituents DNAN and NTO in Rana pipiens tadpoles. Environmental Toxicology and Chemistry 4, 873-879.

Wells, M.J., 1958. Factors Affecting Reactions to Mysis by Newly Hatched Sepia. Behaviour. 13, 96-111.

Wells, M.J., 1964. Tactile discrimination of shape by octopus. The Quarterly Journal of Experimental Psychology 16, 156-162.
Yasumuro, H., Ikeda, Y., 2016. Environmental Enrichment Accelerates the Ontogeny of Cryptic Behavior in Pharaoh Cuttlefish (Sepia pharaonis). Zoological Science 33, 255265.

Yasumuro, H., Ikeda, Y., 2018. Environmental enrichment affects the ontogeny of learning, memory, and depth perception of the pharaoh cuttlefish Sepia pharaonis. Zoology 128, 27-37.

Zepeda, E.A., Veline, R.J., Crook, R.J., 2017. Rapid Associative Learning and Stable Long-Term Memory in the Squid Euprymna scolopes. The Biological Bulletin 232, 212-218.

Zoratto, F., Cordeschi, G., Grignani, G., Bonnani, R., Alleva, E., Nascetti, G., Mather, J., Carere, C., 2018. Variability in the "stereotyped" prey capture sequence of male cuttlefish (Sepia officinalis) could relate to personality differences. Animal Cognition 2, 773-785.

Zylinski, S., Darmaillacq, A.S., Shashar, N., 2012. Visual interpolation for contour completion by the European cuttlefish (Sepia officinalis) and its use in dynamic camouflage. Proceedings of the Royal Society B: Biological Sciences 279, 2386-2390.

Publisher's note Springer Nature remains neutral with regard to jurisdictional claims in published maps and institutional affiliations. 